Portland State University

PDXScholar

7-28-2008

\title{
Lidar System Model for Use With Path Obscurants and Experimental Validation
}

J. W. Giles

Portland State University

I. N. Bankman

R. M. Sova

T. R. Morgan

Donald D. Duncan

Portland State University

See next page for additional authors

Follow this and additional works at: https://pdxscholar.library.pdx.edu/ece_fac

Part of the Electrical and Computer Engineering Commons Let us know how access to this document benefits you.

\section{Citation Details}

J. W. Giles, I. N. Bankman, R. M. Sova, T. R. Morgan, D. D. Duncan, J. A. Millard, W. J. Green, and F. J. Marcotte, "Lidar system model for use with path obscurants and experimental validation," Appl. Opt. 47, 4085-4093 (2008)

This Article is brought to you for free and open access. It has been accepted for inclusion in Electrical and Computer Engineering Faculty Publications and Presentations by an authorized administrator of PDXScholar. Please contact us if we can make this document more accessible: pdxscholar@pdx.edu. 


\section{Authors}

J. W. Giles, I. N. Bankman, R. M. Sova, T. R. Morgan, Donald D. Duncan, J. A. Millard, W. J. Green, and F. J. Marcotte

This article is available at PDXScholar: https://pdxscholar.library.pdx.edu/ece_fac/123 


\title{
Lidar system model for use with path obscurants and experimental validation
}

\author{
J. W. Giles, ${ }^{1, \star}$ I. N. Bankman, ${ }^{1}$ R. M. Sova, ${ }^{1}$ T. R. Morgan, ${ }^{2}$ D. D. Duncan, ${ }^{3}$ \\ J. A. Millard, ${ }^{4}$ W. J. Green, ${ }^{5}$ and F. J. Marcotte ${ }^{1}$ \\ ${ }^{1}$ The Johns Hopkins University Applied Physics Laboratory, 11100 Johns Hopkins Road, Laurel, Maryland 20723, USA \\ ${ }^{2} 1603$ Azalea Dr., Cedar Park, Texas 78613, USA \\ ${ }^{3}$ Oregon Health and Science University, Department of Biomedical Engineering, 3303 Southwest Bond Avenue, \\ Portland, Oregon 97239, USA \\ ${ }^{4}$ Raytheon Missile Systems, P.O. Box 11337, Building 801, Mail Station H15, Tucson, Arizona 85734, USA \\ ${ }^{5}$ NASA/Goddard Space Flight Center, Northrop Grumman Technical Services, Code 429, \\ Building 16W, Room N240R, Greenbelt, Maryland 20771, USA \\ *Corresponding author: john.giles@jhuapl.edu \\ Received 6 May 2008; accepted 19 May 2008; \\ posted 20 June 2008 (Doc. ID 95578); published 28 July 2008
}

\begin{abstract}
When lidar pulses travel through a short path that includes a relatively high concentration of aerosols, scattering phenomena can alter the power and temporal properties of the pulses significantly, causing undesirable effects in the received pulse. In many applications the design of the lidar transmitter and receiver must consider adverse environmental aerosol conditions to ensure the desired performance. We present an analytical model of lidar system operation when the optical path includes aerosols for use in support of instrument design, simulations, and system evaluation. The model considers an optical path terminated with a solid object, although it can also be applied, with minor modifications, to cases where the expected backscatter occurs from nonsolid objects. The optical path aerosols are characterized by their attenuation and backscatter coefficients derived by the Mie theory from the concentration and particle size distribution of the aerosol. Other inputs include the lidar system parameters and instrument response function, and the model output is the time-resolved received pulse. The model is demonstrated and experimentally validated with military fog oil smoke for short ranges (several meters). The results are obtained with a lidar system operating at a wavelength of $0.905 \mu \mathrm{m}$ within and outside the aerosol. The model goodness of fit is evaluated using the statistical coefficient of determination whose value ranged from 0.88 to 0.99 in this study. (C) 2008 Optical Society of America
\end{abstract}

OCIS codes: $\quad 280.3400,280.3640,290.1090,290.1350$.

\section{Introduction}

Lidar is often used for range finding [1]. A major issue with the use of this type of system in obscurants such as clouds, fog, or battlefield conditions is that the lidar return from the obscurant can be significantpotentially greater than the return from a solid object

0003-6935/08/224085-09\$15.00/0

(C) 2008 Optical Society of America (hard target). This effect can cause confusion when the lidar is used to detect a hard target in applications such as smart munitions, sensors, and vehicle and maritime vessel collision avoidance in the presence of obscurants such as clouds, fog, and battlefield smoke [2]. To analyze this phenomenon further, a model has been developed to calculate the actual time-resolved return from the obscurant and the hard target at ranges down to several meters. The model is able to predict the transimpedance amplifier output waveform used in some lidar systems operating in 
various atmospheric conditions. The model addresses the situations where the lidar is operating in clear air, where the lidar and the target are within an obscurant, and when the lidar is outside the obscurant.

An empirically determined impulse response function from a commercial short-range system that operates at a wavelength of $0.905 \mu \mathrm{m}$ is used in the prediction of the analog output waveform for this particular system. Computed waveforms show the effects of backscatter for aerosol fog oil smoke conditions. Experimental validation of the model for a hard target in military fog oil smoke at close range is shown. The model equations are presented in Section 2 . The modeling of the smoke conditions is presented in Section 3. The experimental configuration and conditions in the smoke chamber are discussed in Section 4. The results showing the comparison of the theoretical calculations with the experimental results with the fog oil smoke are presented in Section 5 .

\section{Equations for Modeling the Time-Resolved Lidar Return Waveform}

Figure 1 shows the case where the sensor is outside and the hard target is within the obscurant whose

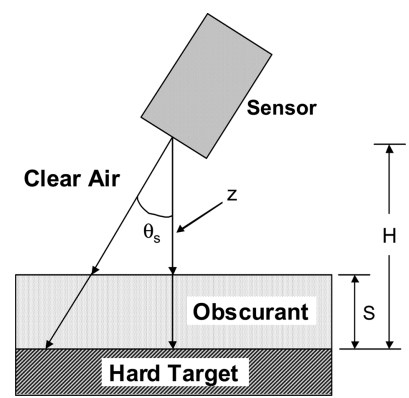

Fig. 1. Configuration for the sensor located outside the obscurant.

quate for the cases treated in this paper. The single scattering equations that describe our situation are given in Subsection 2.A.

\section{A. Return Laser Power}

Considering single scattering, the return laser power from a Lambertian target [3-5] larger than or equal to the beamwidth for all $\theta_{s}$ is given by

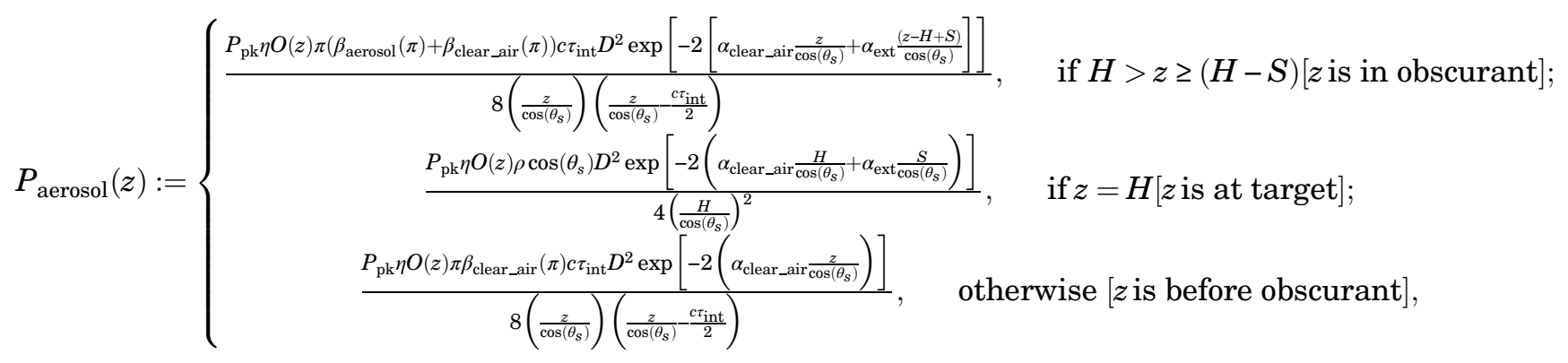

thickness is $S$, and the distance from the target to the sensor is $H$ with an angle of $\theta_{s}$ between the sensor axis and the target normal. The concentration of the obscurant can vary from very thin to very thick. As the obscurant becomes thicker, the multiple scattering of the lidar return becomes more significant. For light to medium thicknesses, single scattering lidar equations can be used. As the obscurant becomes thicker, the single scattering equations become less valid. For the short path lengths of interest in this work, the single scattering treatment is adequate in most of the cases. As the obscurant becomes thicker, multiple scattering issues must be considered. We start our discussion with the simpler single scattering treatment. Later (in Subsection 3.C) we present a general discussion of multiple scattering involving the obscurants. In Subsection 5.B we show that the single scattering treatment appears to be ade- where $P_{\text {aerosol }}(z)$ is the optical power returned from distance $z$ (measured from the sensor) of the obscuranttarget system $(\mathrm{W}), \tau_{\text {int }}$ is the integration time of lidar system $(\mathrm{s}) \approx$ laser transmitter pulse + width receiver response time, $P_{\mathrm{pk}}$ is the peak laser power equal to the pulse energy $/ \tau_{\text {int }}(\mathrm{W}), \eta$ is the optical transmittance of the system (dimensionless), $O(z)$ is the overlap function (dimensionless), $\beta_{\text {aerosol }}(\pi)$ is the volume scattering function of the aerosol in the backward direction $\left(\mathrm{m}^{-1} \mathrm{sr}^{-1}\right), \beta_{\text {clear_air }}(\pi)$ is the volume scattering function of clear air in the backward direction $\left(\mathrm{m}^{-1} \mathrm{sr}^{-1}\right), \rho$ is the hemispherical reflectance of the extended Lambertian target (dimensionless), $c$ is the speed oflight $\left(3 \times 10^{8} \mathrm{~m} / \mathrm{s}^{-1}\right), D$ is the receiver aperture diameter $(\mathrm{m}), \alpha_{\text {clear_air }}$ is the extinction coefficient of clear air $\left(\mathrm{m}^{-1}\right), \alpha_{\text {ext }}$ is the extinction coefficient of the aerosol $\left(\mathrm{m}^{-1}\right)$, and $\theta_{s}$ is the angle between the sensor axis and the target normal. 
If the obscurant covers both the sensor and the target, the above equations become series waveform is a realistic output waveform for a single pulse. If the response time of the sensor is

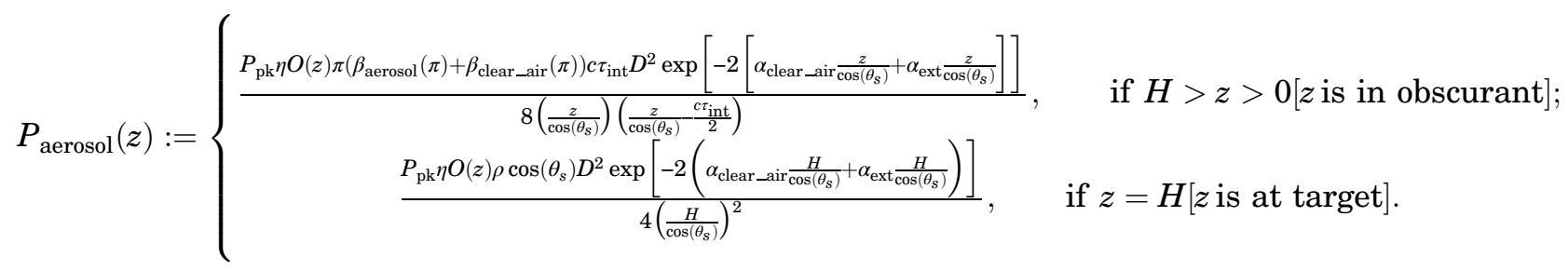

These equations yield the received laser power as a function of distance, $z$, measured from the sensor and equal to $H$ at the hard target. The $c \tau_{\text {int }} / 2$ term in the denominator of Eqs. (1) and (2) is the result of considering the output laser pulse from the detector as a square wave with an effective pulse width of $\tau_{\text {int }}$ [3]. To use these equations without modification, $c \tau_{\text {int }} / 2$ should be small compared with $H$ and $S$ in Fig. 1. However, a technique is shown in Subsection 2 .B for using these equations with larger values of $c \tau_{\text {int }} / 2$. Distance $z$ can be converted to the elapsed time, $t$, by substituting $z=c t / 2$. These equations provide the laser power as a function of the range of the laser pulse leading edge from the sensor or, equivalently, the elapsed time. Therefore from Eqs. (1) and (2), we can calculate the return from a target when the sensor is outside the aerosol and only the target is within the obscurant, and also for the case in which both the target and the sensor are immersed in the obscurant. The power received by the sensor operating in or near an obscurant, such as a cloud, without a hard target present can also be calculated.

\section{B. Instrument Function Effects}

The following discussion denotes $F(t)=P_{\text {aerosol }}(t)$ as the aerosol-target signature function, i.e., impulse response, where $P_{\text {aerosol }}(t)$ is obtained from $P_{\text {aerosol }}(z)$ using $z=c t / 2$ for the change of variable.

If the laser pulse width is very narrow, say $\tau \sim 1 \mathrm{~ns}$, and the detector and receiver have a very short response time, say, $\tau_{R} \sim 0.1 \mathrm{~ns}$ (receiver bandwidth $\left.B=1 /\left(2 \tau_{R}\right) \sim 5 \mathrm{GHz}\right)$, so the instrument response approximates a delta function, Eqs. (1) and (2) can be used to convert directly from the optical power to the actual voltage output of the electronic amplifier (as a function of time). This conversion is expressed as

$$
F^{\prime}(t)=F(t) \Re G,
$$

where $\Re$ is the responsivity of the detector $(\mathrm{A} / \mathrm{W})$, and $G$ is the amplifier gain (V/A). The calculated time somewhat longer (i.e., if the laser pulse is somewhat wider or the detector response time is somewhat longer), these equations do not represent a realistic output waveform. In these cases we then need to determine the effect of lidar system parameters, such as transmitter pulse width and detector response time, on the return waveform. Here the time-resolved output of lidar $f(t)$ is given by

$$
f(t)=\int_{0}^{t^{\prime}=t} I\left(t^{\prime}\right) F^{\prime}\left(t-t^{\prime}\right) \mathrm{d} t^{\prime}=I(t) * F^{\prime}(t)
$$

where $I(t)$ is the instrument impulse response function, $F^{\prime}(t)$ is the function in Eq. (3) above, and * denotes convolution. $I(t)$ can be calculated using system parameters. The impulse response can also be determined by pointing the transmitter directly into the receiver in clear air, if feasible, or by directing the return beam into the receiver using a reflective target in clear air. The instrument impulse response function is then convolved with $F^{\prime}(t)$ to calculate the timeresolved output of the lidar. If the area under the instrument impulse response function is normalized to unity, the convolution gives the actual voltage output of the system. If this is not done or the parameters in Eq. (3) are not well known, the actual output is known to within a scaling constant after taking the convolution.

From our estimate of the responsivity of the detector $(0.65$ to $1.0 \mathrm{~A} / \mathrm{W})$ and the amplifier gain (5000 to $10,000 \mathrm{~V} / \mathrm{A}$ ), we estimate the product $\Re G$ in Eq. (3) to be $\sim 3000$ to $10,000 \mathrm{~V} / \mathrm{W}$. Because of this, after taking the convolution with the area under the instrument impulse response function normalized to unity, the output is known to within a factor of $\sim 3$. From Eq. (3) this range of values for $\Re G$ along with our calculation of $F(t)$ gives an estimate of the peak output voltage that brackets the experimentally observed peak values. As discussed in Subsection 5.A in the experimental analysis, a scaling constant $\bar{K}$, which is 
equal to $\Re G$, was selected so the modeled peak output agreed with the experimental peak receiver output.

\section{Modeling of Smoke Conditions}

The obscurant used in this research was military fog oil smoke since it is easily generated, stable and long lasting, and reasonably nontoxic.

\section{A. Particle Size Distribution}

The fog oil smoke particle size distribution, $n(r)$, is generally approximated by a lognormal distribution

$$
n(r)=\frac{\mathrm{d} N}{\mathrm{~d} r}=\left[\frac{N}{r \sqrt{2 \pi} \ln \sigma_{g}}\right] \exp \left\{-\frac{\left(\ln r / r_{g}\right)^{2}}{2\left(\ln \sigma_{g}\right)^{2}}\right\},
$$

where $r$ is the particle radius $(\mu \mathrm{m}), N$ is the particle number density (number $\left./ \mathrm{m}^{3}\right), r_{g}$ is the distribution median radius $(\mu \mathrm{m})$, and $\sigma_{g}$ is the distribution width parameter (dimensionless).

In this distribution the natural logarithm of the particle radius rather than the particle radius itself is normally distributed $[\underline{6}, 7]$. The mass median diameter, MMD, often used in the literature [윽] is given by

$$
\ln (\mathrm{MMD})=\ln 2 r_{g}+3\left(\ln \sigma_{g}\right)^{2} .
$$

The parameters for our lognormal fog oil smoke particle distribution are $\mathrm{MMD}=1.26 \mu \mathrm{m}, \sigma_{g}=1.4$, and $r_{g}=0.45 \mu \mathrm{m}$.

\section{B. Mie Calculations}

Because fog oil smoke particles are spherical and their size is on the order of the wavelength of the radiation [8], Mie theory is applicable. Theoretical calculations using code [9] based on the Mie theory [10] were performed to determine the values of the extinction coefficient, $\alpha_{\text {ext }}$, and the backscatter coefficient, $\beta_{\text {aerosol }}(\pi)$, for various smoke conditions.

The extinction cross section per unit volume calculated from the Mie theory is given by

$$
\alpha_{\mathrm{ext}}=\int_{0}^{\infty} C_{\mathrm{ext}}(r) n(r) \mathrm{d} r,
$$

where $C_{\text {ext }}$ is the extinction cross section $\left(\mathrm{m}^{2}\right)$. We further write

$$
\alpha_{\mathrm{ext}}=\kappa_{\mathrm{ext}} C,
$$

where $\kappa_{\text {ext }}$ is the mass extinction coefficient that is particle distribution dependent $\left(\mathrm{m}^{2} / \mathrm{g}\right), C$ is the aerosol mass concentration $\left[\int_{0}^{\infty} \frac{4}{3} \pi r^{3} \rho n(r) \mathrm{d} r\left(\mathrm{~g} / \mathrm{m}^{3}\right)\right]$, and $\rho$ is the density of the fog oil droplets comprising the aerosol cloud $\left(0.89 \mathrm{~g} / \mathrm{cm}^{3}\right)$.

For a lognormal distribution the mass concentration is given by [11]

$$
C=\frac{4}{3} \pi r_{g}^{3} \exp \left[\frac{9}{2}\left(\ln \sigma_{g}\right)^{2}\right] \rho N .
$$

Using the value of $\alpha_{\text {ext }}$ from Eq. (7) normalized for a number concentration of 1 particle $/ \mathrm{m}^{3}$ by dividing the right-hand side of Eq. (7) by $N=\int_{0}^{\infty} n(r) \mathrm{d} r$ and the value of $C$ in Eq. (9) for $N=1$ particle $/ \mathrm{m}^{3}$, we can solve for $\kappa_{\text {ext }}$ in Eq. (8). Then, again with Eq. (8), we can solve for $\alpha_{\text {ext }}$ for a given concentration.

Similarly the scatter cross section per unit volume calculated from Mie theory is expressed as

$$
\alpha_{\mathrm{sca}}=\int_{0}^{\infty} C_{\mathrm{sca}}(r) n(r) \mathrm{d} r
$$

where $C_{\text {sca }}$ is the scatter cross section $\left(\mathrm{m}^{2}\right)$, and

$$
\alpha_{\mathrm{sca}}=\kappa_{\mathrm{sca}} C,
$$

where $\kappa_{\text {sca }}$ is the mass scatter coefficient.

Now we calculate $\beta_{\text {aerosol }}(\pi)$ for the desired lognormal distribution. The phase function [9] is given by

$$
P(\theta)=N \frac{4 \pi S_{11}(\theta)}{k^{2} \alpha_{\text {sca }}},
$$

where $S_{11}(\theta)$ is the first Mueller matrix element calculated from the Mie theory, and

$$
k=2 \pi / \lambda,
$$

where $\lambda$ is the wavelength. Finally with

$$
\beta_{\text {aerosol }}(\pi)=\frac{P(\pi)}{4 \pi} \alpha_{\mathrm{sca}}=\frac{P(\pi)}{4 \pi} \kappa_{\mathrm{sca}} C,
$$

we calculate $\beta_{\text {aerosol }}(\pi)$ if we know the concentration, $C$.

The complex index of refraction for fog oil smoke [12] at a wavelength of $0.905 \mu \mathrm{m}$ is $1.4743+i 0.0000 \overline{02}$. The calculated parameter values for light, medium, heavy, and very heavy fog oil smoke with the lognormal parameters above are shown in Table 1 . These values may be entered into Eqs. (1) and (2) to calculate the waveforms for the various fog oil smoke conditions. The definitions of the various smoke densities are similar to those in Ref. [13]. The $\alpha_{\text {clear_air }}$ and $\beta_{\text {clear_air }}(\pi)$ for clear air used in these calculations are $1.2 \times$ $10^{-4} \mathrm{~m}^{-1}$ and $1.3 \times 10^{-7} \mathrm{~m}^{-1}-\mathrm{sr}^{-1}$, respectively.

\section{Single and Multiple Scattering}

The intensity of multiply scattered light depends on the scattering medium properties, such as the size and distribution of the scattering particles, and the optical depth of the scattering volume given by

$$
\tau_{\mathrm{OD}}=\int_{0}^{Z} \alpha_{\text {ext }}(z) \mathrm{d} z .
$$

The amount of multiply scattered light also depends significantly on the lidar geometry, increasing dramatically with increasing laser beam divergence, the receiver's field of view, and the distance between the lidar and the scattering volume [14]. If $\tau_{\mathrm{OD}}<0.8$, 
Table 1. Fog Oil Smoke Parameters Calculated from the Mie Theory

\begin{tabular}{ccc}
\hline Smoke Concentration $\left(\mathrm{g} / \mathrm{m}^{3}\right)$ & $\alpha_{\text {ext }}\left(\mathrm{m}^{-1}\right)$ & $\beta_{\text {aerosol }}(\pi)\left(\mathrm{m}^{-1} \mathrm{sr}^{-1}\right)$ \\
\hline Light $(0.0152)$ & 0.072 & $1.58 \times 10^{-3}$ \\
Medium $(0.038)$ & 0.18 & $3.95 \times 10^{-3}$ \\
Heavy $(0.076)$ & 0.36 & $7.90 \times 10^{-3}$ \\
Very heavy $(0.114)$ & 0.54 & $1.19 \times 10^{-2}$ \\
\hline
\end{tabular}

single scattering prevails; for $0.8<\tau_{\mathrm{OD}} \leq 1$, there is only a small contribution from second-order scattering. For larger values of the optical depth, higher orders of multiple scattering should be considered [14]. For medium smoke and path lengths of 2 to $4 \mathrm{~m}$, for which $\alpha_{\text {ext }}=0.18 \mathrm{~m}^{-1}$ from Table 1 , the optical depth is $\sim 0.3$ to 0.6 , so multiple scattering is not significant. For the heavy smoke for which $\alpha_{\text {ext }}=0.36 \mathrm{~m}^{-1}$ from Table 1, the optical depth is 0.72 to 1.4 for path lengths of 2 to $4 \mathrm{~m}$, and we may start to see some multiple scattering effects.

\section{Measurement of Time-Resolved Lidar Output in Fog Oil Smoke}

\section{A. Description of Lidar Used in Smoke Tests}

The time-resolved return pulses of a short-range lidar were measured in a fog oil smoke chamber in various fog oil smoke conditions. Table 2 shows the major system parameter values of the lidar system (H. N. Burns Engineering Corporation, Orlando, Florida) that is based on a GaAs laser diode and a silicon PIN diode.

The effects of the transmitter pulse width, as well as the receiver response time, are characterized with the impulse response function for this sensor as shown in Fig. 2. This function was determined experimentally using a planar reflective target placed in a position perpendicular to the laser beam in clear air as discussed in Subsection 2.B. The curve, as shown in Fig. 2, is normalized to have a peak value of unity. The full width at half maximum (FWHM)

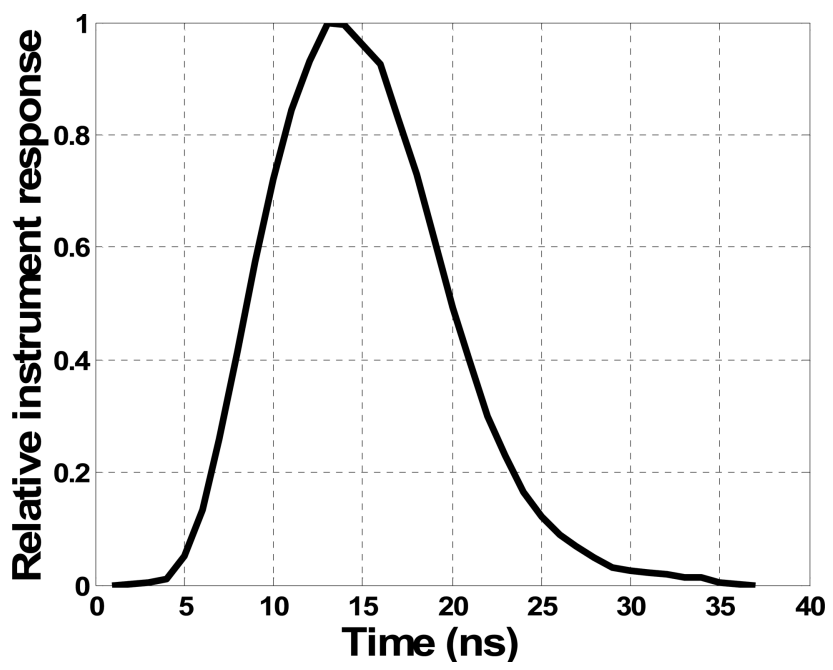

Fig. 2. Instrument response function for the short-range lidar. value of the impulse response function is approximately $12 \mathrm{~ns}$.

\section{B. Description of Smoke Chamber}

The smoke chamber, shown in Fig. 3 , is a $12.2 \mathrm{~m} \times$ $2.1 \mathrm{~m} \times 2.1 \mathrm{~m}$ portable shipping container with a Plexiglas window. Two fans, one near the rear and one near the front of the chamber, were used to help disperse the smoke evenly. The sensor and motorized track assembly can be placed inside or outside the smoke chamber. The output voltage of the receiver's transimpedance amplifier was recorded with a Tektronix TDS 754D digital oscilloscope, which has a maximum digitization rate of $2 \mathrm{GHz}$.

\section{Description of Fog Oil Smoke and Smoke Generator}

The smoke was generated by vaporizing military standard grade fuel number 2 (SGF2) fog oil with a Neutralizer model 2760 thermal smoke generator (Curtis Dyna-Fog, Ltd., Westfield, Indiana). Measurements of particle size distribution were made with a model 100 particle measuring systems forward-scattering spectrum probe [15] (also known as a Knollenberg particle counter) manufactured by Particle Measuring Systems, Inc. of Boulder, Colorado. This instrument measures the particle diameters in 15 bins, each $0.5 \mu \mathrm{m}$ wide starting with diameters of 0.5 to $1.0 \mu \mathrm{m}$ for the first bin, 1.0 to $1.5 \mu \mathrm{m}$ for the second, up to 7.5 to $8.0 \mu \mathrm{m}$ for the fifteenth bin creating the distribution shown in Fig. 4. The size of the measurement bins (as radii) is indicated by the horizontal bars on the graph. For comparison a lognormal distribution is also shown on the graph. Our best estimate of the particle size distribution from the provider of the smoke generator [12] and these data is $\mathrm{MMD}=1.26 \mu \mathrm{m}, \sigma_{g}=1.4$, and $r_{g}=0.45 \mu \mathrm{m}$. Note that the number of particles in the upper tail is greater than that of the lognormal distribution.

\section{Measurement of Smoke Concentration and Effects of Particle Size Distribution}

The density of the smoke was determined using a $\mathrm{He}-\mathrm{Ne}$ laser transmissometer (see Fig. 3) whose receiver was equipped with a chopper, a lock-in amplifier, and a PIN diode. In the case of a highly

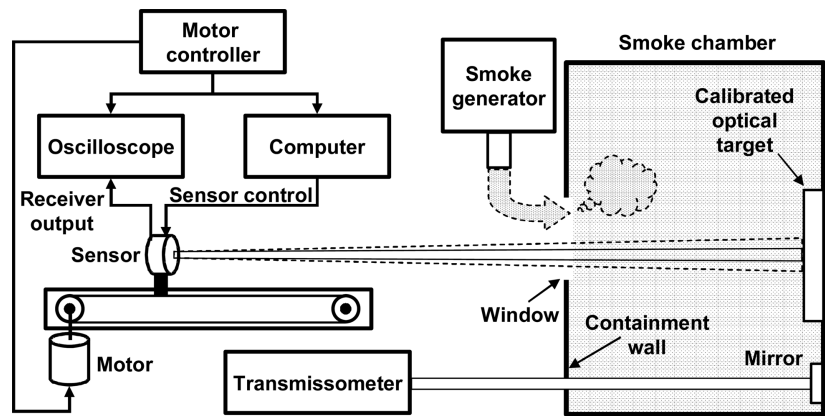

Fig. 3. Fog oil smoke chamber. The sensor and motorized track assembly can also be placed inside the smoke chamber. 


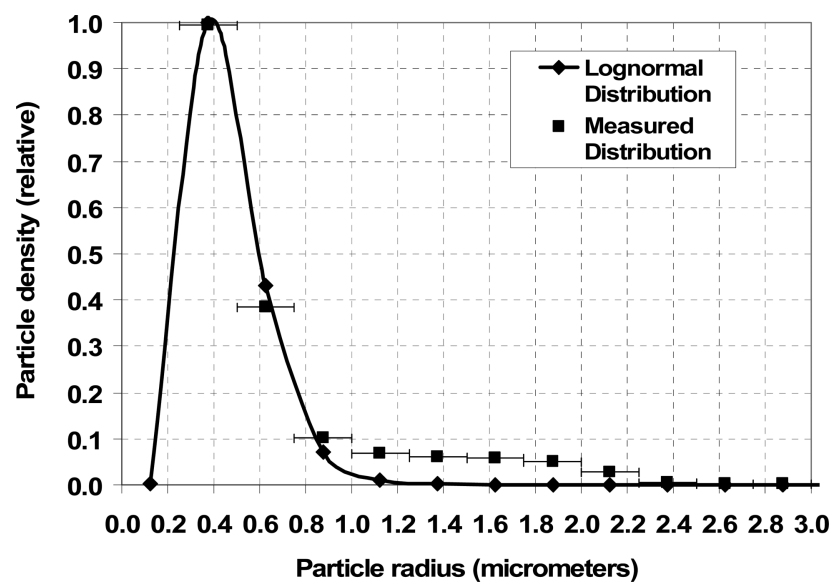

Fig. 4. Particle density versus particle radius for a lognormal distribution with $\mathrm{MMD}=1.26 \mu \mathrm{m}, r_{g}=0.45 \mu \mathrm{m}$, and $\sigma_{g}=1.4$ and the measured distribution. The horizontal bars show bin width ( $0.25 \mu \mathrm{m}$ radius).

collimated beam, the transmittance through smoke is expressed by

$$
T=\exp \left(-\kappa_{\text {ext }} C L\right)
$$

where $\kappa_{\text {ext }}$ is the mass extinction coefficient $\left(\mathrm{m}^{2} / \mathrm{g}\right), C$ is the aerosol droplet mass concentration $\left(\mathrm{g} / \mathrm{m}^{3}\right)$, and $L$ is the total path length in smoke $(\mathrm{m})$.

$\kappa_{\text {ext }}$ depends on the size distribution of particles in the smoke. Figure 5 shows the mass extinction coefficient calculated with the Mie theory for MMD values of $0.25,0.5,1.0$, and $1.5 \mu \mathrm{m}$, and $\sigma_{g}=1.4$. The concentration can be determined using the measured transmittance, $T$, calculated as the ratio of the received laser power in clear air to that through a known smoke type and distance. At a wavelength of $0.6328 \mu \mathrm{m}, \kappa_{\text {ext }}$ is $4.46 \mathrm{~m}^{2} / \mathrm{g}$ for our fog oil smoke with $\mathrm{MMD}=1.26 \mu \mathrm{m}$ and $\sigma_{g}=1.4$.

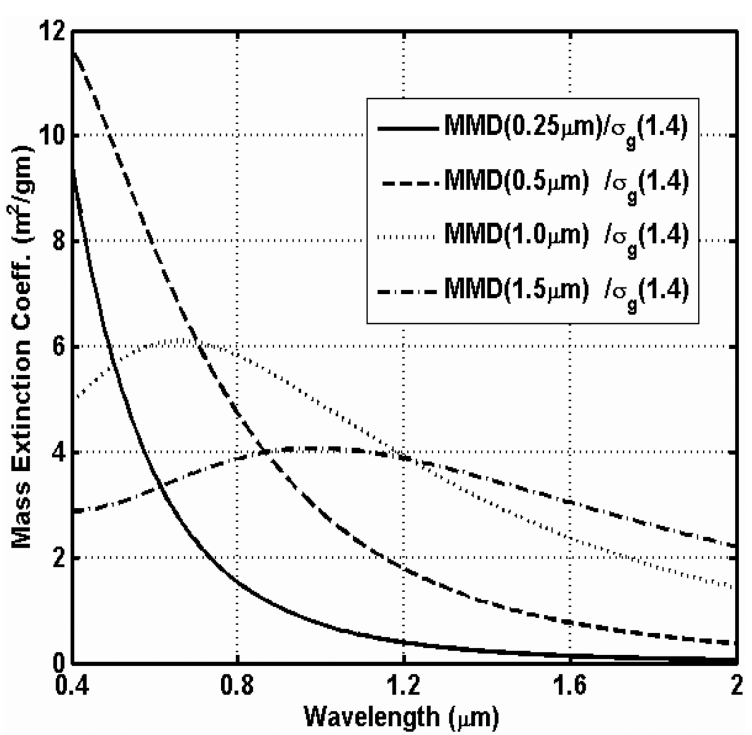

Fig. 5. Mass extinction coefficient versus wavelength for various mass median fog oil smoke diameter particles for a lognormal distribution.
With the known transmittance, path length, and $\kappa_{\text {ext }}$, the smoke concentration can be determined from Eq. (16). For our configuration a mirror was used to double the transmissometer path as shown in Fig. 3. The one-way path length was $\sim 6 \mathrm{~m}$. Uncertainties in the smoke concentrations are estimated to be $\pm 20 \%$. The uncertainties in the smoke concentrations are due to the uncertainty in $\kappa_{\text {ext }}$, estimated to be $\pm 15 \%$, which is caused by the uncertainty in the particle size distribution and the uncertainty in the transmission measurement, which is estimated to be $\pm 15 \%$. These uncertainties combine to give a $\pm 20 \%$ total uncertainty (root sum of squares).

\section{Results}

\section{A. Model and Experimental Comparisons}

Waveforms at $0.905 \mu \mathrm{m}$ calculated from the model for the target and sensor immersed in medium smoke and those measured in the smoke chamber are compared in Fig. 6 . The model calculations were made by calculating $P_{\text {aerosol }}(t)$ with the sensor system parameters in Table 2, and the integration time of the system, $\tau_{\text {int }}$, set equal to $1 \mathrm{~ns}$ in Eq. (2) and then convolving the result with the instrument response function in $1 \mathrm{~ns}$ increments as shown in Fig. 2 using Eq. (4) as discussed in Subsection 2.B. After taking the convolution, the output is known to within a scaling constant because $\Re G$ in Eq. (3) is only known to within a factor of $\sim 3$ for this system. A scaling constant $K$ of $\sim 3000 \mathrm{~V} / \mathrm{W}$, which is equal to $\Re G$, was selected so the modeled peak output agreed with the experimental peak receiver output for the medium smoke case for the sensor-target distance of $2.4 \mathrm{~m}$. The same value of $K$ was also used for the other medium smoke distances.

For the initial calculations in medium smoke, the parameters calculated from the Mie theory are
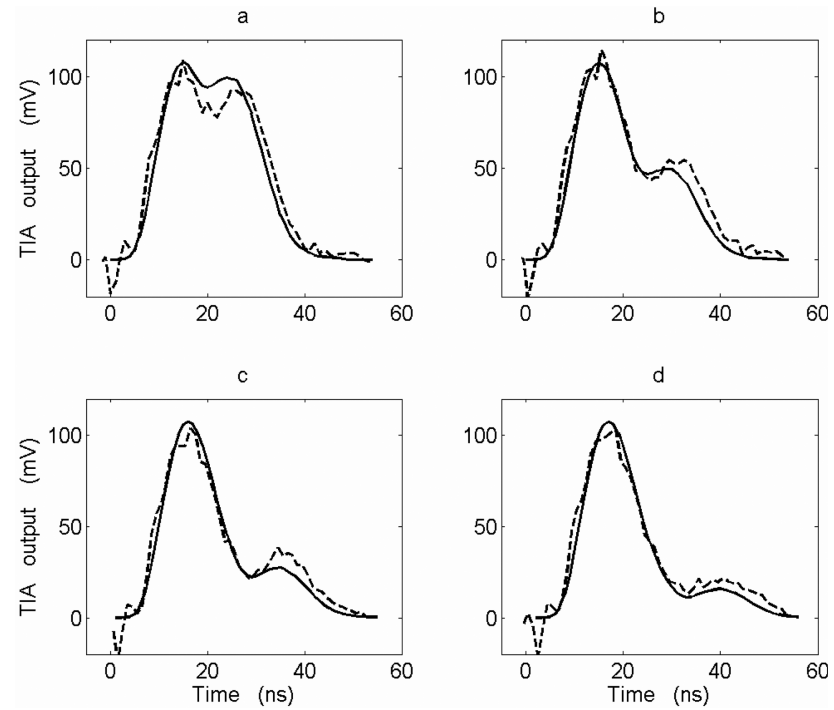

Fig. 6. Model and experimental waveforms for sensor (a) 2.4 (b) 3.0, (c) 3.7, and (d) $4.3 \mathrm{~m}$ from target. The target and the sensor are immersed in medium smoke. The solid curves are model calculations, and the dashed curves are experimental. 
Table 2. Sensor System Parameters

\begin{tabular}{ll}
\hline \multicolumn{1}{c}{ Parameter } & \multicolumn{1}{c}{ Value } \\
\hline Wavelength & $\lambda=0.905 \mu \mathrm{m}$ \\
Pulse energy & $Q=0.64 \mu \mathrm{J}$ \\
Laser pulse width (FWHM) & $\tau=8 \mathrm{~ns}$ \\
Detector response time (rise time) & $\tau_{r}=3.5 \mathrm{~ns}$ \\
Peak power & $P_{\mathrm{pk}}=Q /\left(\tau+\tau_{r}\right)=56 \mathrm{~W}$ \\
Pulse repetition frequency & $5 \mathrm{kHz}$ \\
Amplifier bandwidth & $B=140 \mathrm{MHz}$ \\
Receiver aperture diameter & $D=0.025 \mathrm{~m}$ \\
Transmitter beam spread & $\approx 1^{\circ}$ \\
Receiver field of view & $\approx 8^{\circ}$ \\
Overlap function & $0.2($ dimensionless $)$ \\
0.3 m from sensor & 0.5 \\
$0.45 \mathrm{~m}$ from sensor & 1 \\
Beyond 0.6 m from sensor & \\
\hline
\end{tabular}

shown in Table 1 . We then find a single medium smoke value of $\beta_{\text {aerosol }}(\pi), \alpha_{\text {ext }}$, and the target reflectance to obtain the best-fit visual inspection agreement between the model and experimental results for the four medium smoke distances. These values were adjusted within the estimated uncertainties. Target reflectances were measured relative to Spectralon by comparing return signals from the sensor. The uncertainty in the reflectance measurements for the $0.6 \mathrm{~m} \times 0.9 \mathrm{~m}$ paper-covered polystyrene board gray and black targets is estimated to be $\pm 25 \%$. There is an estimated uncertainty of $\pm 25 \%$ in the value of $\alpha_{\text {ext }}$ and $\beta_{\text {aerosol }}(\pi)$ defined by Eqs. (8) and (14) owing to the uncertainty in the smoke concentrations and in the parameters in Eqs. (8) and (14), which depend on the particle size distribution. There is an estimated $\pm 15 \%$ uncertainty because of the uncertainty in the particle size distribution and $\mathrm{a} \pm 20 \%$ uncertainty in the smoke concentration as discussed in Subsection 4.D. These uncertainties combine to give a $\pm 25 \%$ total uncertainty (root sum of squares). The smoke and target parameter values used to obtain the good-fit model curves are shown in Table 3 . The smoke parameter values calculated from the Mie theory for medium smoke shown in Table 1 and the measured values of the smoke concentration and target reflectance are shown in Table 3 for comparison with the good-fit model values. The sensor-target distances for the plots shown in Fig. 6 are 2.4, 3.0, 3.7, and $4.3 \mathrm{~m}$. The fit between model output and experimental data was quantified with the coefficient of determination $R^{2}$ defined as

$$
R^{2}=1-\mathrm{RSS} / \mathrm{TSS},
$$

where RSS is the sum of squares of each residual difference between the model value and the experimental value, $\operatorname{TSS}=N$ times the variance of the experimental data values, and $N$ is the number of data points. $R^{2}$ was 0.956 or better for the plots shown in Fig. 6.

For the waveforms in Fig. 6 , the near return, or first peak, is the return from the smoke and the second is the return from the hard target. The first peak shape is determined by the instrument properties, including the overlap function and the instrument response function, as well as the smoke properties. We see that as the sensor moves farther away from the target, the return from the hard target gets weaker, but the return from the smoke remains the same. The hard target return decreases at longer path lengths because of (1) the high attenuation of the fog oil smoke $\left(\alpha=0.18 \mathrm{~m}^{-1}\right)$ and (2) the $z^{-2}$ dependence of the return as shown in Eq. (2). Since the instrument is immersed in homogeneous fog oil smoke, the smoke return in Fig. 6 does not change as the instrument is moved. The near field return from the fog oil smoke has the larger amplitude as compared with the returns from the hard target.

Using the same method and the same value of $K$ from above, the comparisons of the model and experimental waveforms for the sensor in light smoke and heavy smoke are shown in Figs. $\underline{7}$ and $\underline{8}$, respectively. In Fig. 7 we again see the return from the smoke at near range followed by the hard target return. The hard target return (second peak) is more prominent than in the medium smoke case, because (1) there is less backscatter from the lower density smoke making the smoke peak smaller, and (2) there is less attenuation from the smoke making the hard target peak larger. The coefficients of determination for these comparisons are 0.892 or better. For the heavy smoke condition in Fig. 8 , the hard target cannot be seen at any distance. Once again, for each distance from the target in heavy smoke, we are looking at the same thickness of smoke column (for homogeneous smoke) in front of the transmitter-detector, so the returns for the various distances all look the same. The coefficients of determination for these comparisons are 0.984 or better. The measured and calculated parameters used in the model for these conditions are once again compared with the goodfit model comparisons in Table 3 .

Comparisons of the model and experimental waveforms for the sensor outside of medium smoke are

Table 3. Parameter Comparison for Modeled and Experimental Waveforms ${ }^{a}$

\begin{tabular}{ccccc}
\hline Sensor Configuration & In Light Smoke & In Medium Smoke & In Heavy Smoke & Outside Medium Smoke \\
\hline$C\left(\mathrm{gm} / \mathrm{m}^{3}\right)$ & $0.0152 / 0.0152$ & $0.038 / 0.038$ & $0.076 / 0.076$ & $0.114 / 0.114$ \\
Target reflectance $(\%)$ & $15.2 / 19.0$ & $15.2 / 19.0$ & $15.2 / 15.2$ & $4.0 / 4.0$ \\
$\beta_{\text {aerosol }}(\pi)\left(\mathrm{m}^{-1} \mathrm{sr}^{-1}\right)$ & $(1.58 / 1.32) \times 10^{-3}$ & $(4.0 / 3.3) \times 10^{-3}$ & $(7.9 / 7.9) \times 10^{-3}$ & $(4.0 / 3.3) \times 10^{-3}$ \\
$\alpha_{\text {ext }}\left(\mathrm{m}^{-1}\right)$ & $0.072 / 0.073$ & $0.18 / 0.16$ & $0.36 / 0.36$ & $0.18 / 0.16$ \\
\hline
\end{tabular}

${ }^{a}$ The first number is the calculated or measured value. The second number is the good-fit value used for the model waveform in Figs. $\underline{6}-\underline{9}$. 
a

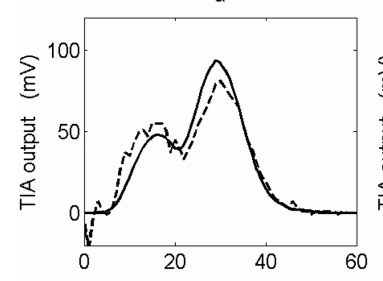

c
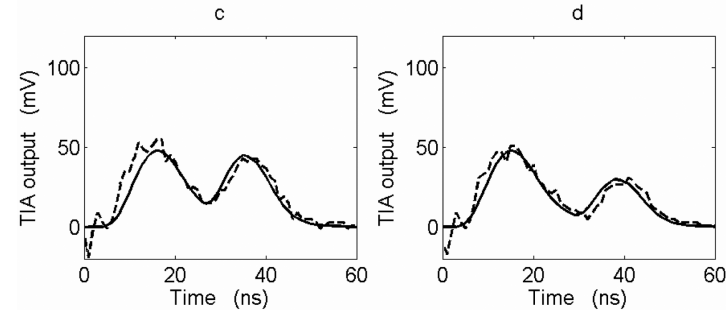

Fig. 7. Model and experimental waveforms for sensor (a) 2.7, (b) 3.0, (c) 3.7, and (d) $4.3 \mathrm{~m}$ from target. The target and the sensor are immersed in light smoke. The solid curves are model calculations, and the dashed curves are experimental.

shown in Fig. 9. The 4\% reflectance target is located $7.3 \mathrm{~m}$ from the smoke interface. In this case only returns from the air-smoke interface are seen; the target is completely obscured. The distance to the hard target in this case has roughly doubled from the previous example (from $\sim 4$ to now $\sim 8 \mathrm{~m}$ ). Because of range dependence $\left(z^{-2}\right)$, the signal return will be approximately four times less. The coefficients of determination for Figs. 9(a)-9(d) are 0.977, 0.936, 0.877, and 0.923 , respectively. The lower signal levels in the experimental curves in Figs. 9(c) and 9(d) reduce the coefficients, owing to lower signal-to-noise ratio. The measured and calculated parameters used in the model for these conditions are once again compared with the good-fit model comparisons in Table 3 .

The agreement between the calculated and experimental waveforms shown in Figs. $6-\underline{9}$ is good with a coefficient of determination of $0.8 \overline{77}$ or higher. From
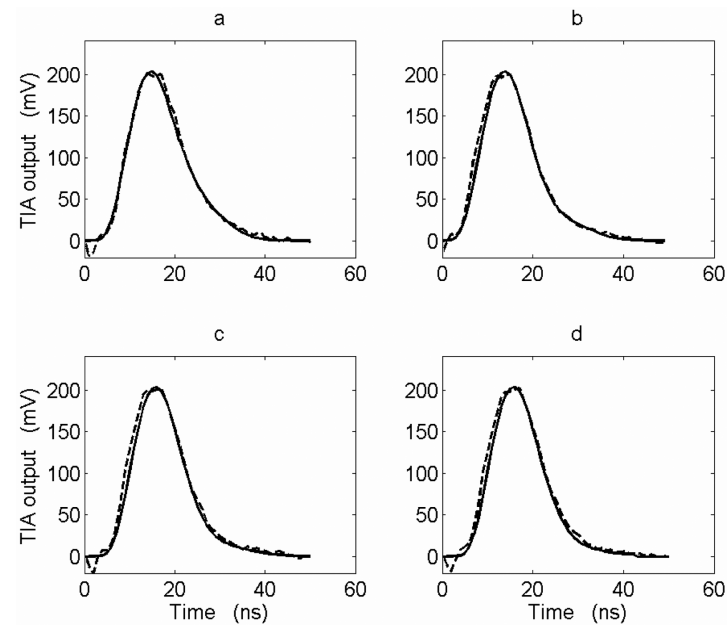

Fig. 8. Model and experimental waveforms for sensor (a) 2.4, (b) 3.0, (c) 3.7, and (d) $4.3 \mathrm{~m}$ from target. The target and the sensor are immersed in heavy smoke. The solid curves are model calculations, and the dashed curves are experimental.
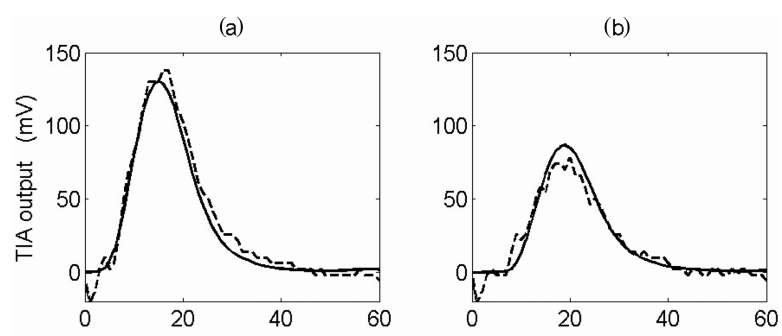

(c)

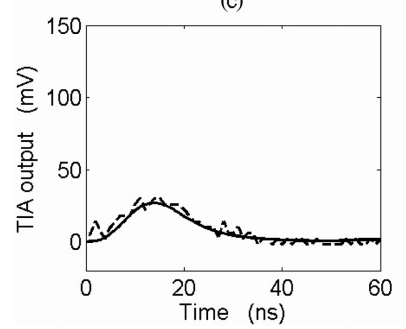

(d)

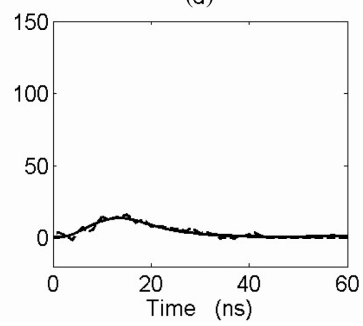

Fig. 9. Model and experimental waveforms for sensor (a) 0, (b) 0.3 , (c) 0.6 , and (d) $1.2 \mathrm{~m}$ from medium smoke interface. The $4 \%$ reflectance target is located $7.3 \mathrm{~m}$ from the smoke interface. The solid curves are model calculations, and the dashed curves are experimental.

Table 3 the calculated and measured parameters agree with the good-fit model parameters to within $25 \%$ or better. It is believed the discrepancies between the good-fit model parameters and those calculated with the Mie theory are due to uncertainty in the smoke concentrations, which are caused by uncertainty in the transmissometer measurements from which the smoke concentrations are derived, and uncertainty in the particle size distribution. The departure of the particle size distribution from lognormal at the larger sizes is shown in Fig. 4. More particles in that size range would lower the calculated backscatter coefficient, thus improving the agreement. Uncertainty in the particle size distribution also results in uncertainty of the smoke concentration, owing to the dependence of $\kappa_{\text {ext }}$, the transmissometer extinction coefficient at $0.6328 \mu \mathrm{m}$, calculated from the Mie theory based on a particular particle size distribution.

\section{B. Discussion of Results}

We see good agreement between the experimental results and the single-scattering predictions. This is an indication that for our geometry, the single-scattering treatment is adequate. As stated previously if the optical depth $\tau_{\mathrm{OD}}<0.8$, single scattering prevails; for $0.8<\tau_{\mathrm{OD}} \leq 1$, there is only a small contribution from second-order scattering. For larger values of the optical depth, higher orders of multiple scattering should be considered [14]. For medium smoke and path lengths of 2 to $4 \overline{\mathrm{m}}$, the good-fit optical depth is $\sim 0.3$ to 0.6 , so multiple scattering is not significant. The comparisons shown in Figs. 6-9 do not indicate that multiple scattering is a major factor. Considering the stretching of the return pulse as an indicator of multiple scattering [16], we do not see significant pulse stretching in the experimental data or significant 
change in the magnitude of the signal due to the extinction coefficient effectively decreasing, because more photons are received at the detector owing to multiple scattering as compared with single scattering. Multiple scattering effects do not appear greater than effects on the waveforms due to uncertainty in the smoke parameters caused by uncertainties in the particle size distribution and smoke concentrations. It is apparent that we are operating just below the regime where multiple scattering would need to be included.

For future work the effects of multiple scattering need to be included in the treatment. Multiplescattering effects would especially be seen at greater ranges (than our $4 \mathrm{~m}$ ). A study might also be conducted for the effects of multiple scattering as the laser beam divergence and receiver field of view is varied.

\section{Conclusions}

A technique is presented for calculating time-resolved lidar return signals at short ranges for a lidar system either inside or outside an obscurant. At short ranges the return from the obscurant can be significant, potentially greater than that from a hard target in the obscurant. The obscurant in this work is military fog oil smoke (SGF2) for which the droplets are spherical and the Mie theory is applicable. Although it is shown that the particle size distribution is not exactly lognormal, such a distribution in which $r_{g}=0.45 \mu \mathrm{m}$, $\sigma_{g}=1.4$, and $\mathrm{MMD}=1.26 \mu \mathrm{m}$ is a reasonably good approximation. The lidar operates at $0.905 \mu \mathrm{m}$. Using the Mie theory, calculations of the extinction coefficient, $\alpha_{\text {ext }}$, and the volume scattering function in the backward direction $\beta_{\text {aerosol }}(\pi)$ at the operational wavelength are shown for light, medium, heavy, and very heavy fog oil smoke concentrations. The ground-truth smoke concentration was calculated using a transmissometer operating at $0.6328 \mu \mathrm{m}$ and applying a mass extinction coefficient based on the Mie theory. Uncertainly in the smoke concentration measurements is estimated to be $\pm 20 \%$.

Comparisons show good agreement between the model and experimental waveforms for short-range sensor distances and for various smoke concentrations. The model accurately predicts the shape of the waveforms. For a lidar and a low-reflectance target immersed in light and medium smoke, two returns can be expected: (1) a backscatter return from the smoke and (2) a return from the hard target. As the density of the smoke increases, only a return from the smoke can be expected because the target is completely obscured due to the attenuation and backscatter of the lidar signal. These results show that a return from the smoke could generate a false target detection if simple thresholding rules are applied. The calculated and measured smoke parameters agree with the good-fit model parameters to within $25 \%$ or better. It is believed these discrepancies are due to uncertainties in the particle size distribution and the departure of the distribution from lognormal at the larger sizes. These, in turn, result in uncertainties in the extinction coefficient used for determining the smoke concentrations and uncertainties in the extinction and backscatter coefficients for the various smoke concentrations. Multiple-scattering effects such as pulse stretching or significantly smaller extinction coefficients are not apparent.

The presented model, validated by experimental results, can be used for short-range calculations if the backscatter and extinction parameters are known for the medium of interest and if the reflectance of the target is known. This model can also be used to predict the performance of the current lidar sensor and other sensors under various atmospheric and battlefield smoke conditions.

The authors gratefully acknowledge the Janney Fellowship from The Johns Hopkins University Applied Physics Laboratory for partial support of this effort.

\section{References}

1. R. W. Byren, "Laser rangefinders," in The Infrared and Electro-Optical Systems Handbook, C. S. Fox, ed. (SPIE Optical Engineering, 1993), Vol. 6, Chap. 2, pp. 77-114.

2. M. Kleiman and N. Shiloah, "The effect of dense atmospheric environment on the performances of laser radar sensors used for collision avoidance," Proc. SPIE 3707, 624-635 (1999).

3. R. M. Measures, Laser Remote Sensing (John Wiley, 1984).

4. H. N. Burns, C. G. Christodoulou, and G. D. Boreman, "System design of a pulsed laser rangefinder," Opt. Eng. 30, 323-329 (1991).

5. G. W. Kamerman, "Laser radar," in The Infrared and ElectroOptical Systems Handbook, C. S. Fox, ed. (SPIE Optical Engineering, 1993), Vol. 6, Chap. 1, pp. 3-76.

6. W. C. Hinds, Aerosol Technology (Wiley, 1999).

7. K. Willeke and P. A. Baron, Aerosol Measurement (Wiley, 1993).

8. H. R. Carlon, D. H. Anderson, M. E. Milham, T. L. Tarnove, R. H. Frickel, and I. Sindoni, "Infrared extinction spectra of some common liquid aerosols," Appl. Opt. 16, 1598-1605 (1977).

9. M. E. Thomas and D. D. Duncan, "Atmospheric transmission," in The Infrared and Electro-Optical Systems Handbook, F. G. Smith, ed. (SPIE Optical Engineering, 1993), Vol. 2, Chap. 1, pp. 1-156.

10. D. Deirmendjian, Electromagnetic Scattering on Spherical Polydispersions (Elsevier, 1969).

11. C. S. Zender, "Particle size distributions: theory and application to aerosols, clouds, and soils," (2008), http://dust.ess.uci .edu/facts/psd/psd.pdf.

12. D. H. Anderson, Soldier Biological Chemical Command (SBCCOM), Edgewood, Maryland 21010 (personal communication, 2001).

13. Joint Technical Coordinating Group for Munitions Effectiveness, Smoke and Aerosol Working Group, "Smoke and natural aerosol parameters (SNAP) manual," Report No. 61JTCG/ ME-85-2 (1985), p. 5-1.

14. V. A. Kovalev and W. E. Eichinger, Elastic Lidar (Wiley, 2004).

15. R. G. Knollenberg, "Techniques for probing cloud microstructure," in Clouds Their Formation, Optical Properties, and Effects, P. V. Hobbs and A. Deepak, eds. (Academic, 1981), pp. $15-91$.

16. R. E. Walker and J. W. McLean, "Lidar equations for turbid media with pulse stretching," Appl. Opt. 38, 2384-2397 (1999). 